\title{
Exploring the Effects of Local Energy Markets on Electricity Retailers and Customers
}

\author{
Dawei Qiu ${ }^{1}$, Yujian $\mathrm{Ye}^{1,2}$, and Dimitrios Papadaskalopoulos ${ }^{1}$ \\ ${ }^{1}$ Department of Electrical and Electronic Engineering, Imperial College London, London, UK \\ ${ }^{2}$ Fetch.ai, Cambridge, UK \\ d.qiu15@imperial.ac.uk, yujian.ye11@imperial.ac.uk, d.papadaskalopoulos08@imperial.ac.uk
}

\begin{abstract}
Local energy markets (LEM) have recently attracted great interest as they enable effective coordination of smallscale distributed energy resources (DER) at the customer side, and avoidance of distribution network reinforcements. However, the introduction of LEM has also significant implications on the strategic interactions between the customers and incumbent electricity retailers. This paper explores for the first time these interactions by proposing a novel multi-period bi-level optimization model, which captures the pricing decisions of a strategic retailer in the upper level (UL) and the response of both independent customers and the LEM (both including flexible consumers, micro-generators and energy storages) in the lower level (LL). Since the $L L$ problem representing the LEM is non-convex, a new analytical approach is employed for solving the developed bi-level problem. The examined case studies demonstrate that the introduction of an LEM reduces the customers' energy dependency on the retailer and limits the retailer's strategic potential of exploiting the customers through large differentials between buy and sell prices. As a result, the profit of the retailer is significantly reduced while the customers, primarily the LEM participants and to a lower extent non-participating customers, achieve significant economic benefits.
\end{abstract}

Index Terms-Bi-level optimization, distributed energy resources, local energy markets, electricity retailers.

\section{NOMENCLATURE}

\section{A. Indices and Sets}

$t \in T \quad$ Time periods

$i \in I \quad$ Flexible consumers (FC) not participating in the LEM

$j \in J \quad$ Micro-generators (MG) not participating in the LEM

$k \in K \quad$ Energy storages (ES) not participating in the LEM

$i^{\prime} \in I^{\prime} \quad$ FC participating in the LEM

$j^{\prime} \in J^{\prime} \quad$ MG participating in the LEM

$k^{\prime} \in K^{\prime}$ ES participating in the LEM

B. Parameters

$\lambda_{t}^{w} \quad$ Wholesale price at period $t(£ / \mathrm{MWh})$

$\lambda^{\text {min }} \quad$ Minimum limit of retail price $(£ / M W h)$

$\lambda^{\max } \quad$ Maximum limit of retail price $(£ / \mathrm{MWh})$

$l_{i, t}^{D} \quad$ Linear benefit coefficient of FC $i$ at period $t$ ( $£ / \mathrm{MWh}$ )

$q_{i, t}^{D} \quad$ Quadratic benefit coefficient of FC $i$ at period $t$ $\left(£ / \mathrm{MWh}^{2}\right)$

$d_{i, t}^{\max } \quad$ Maximum demand limit of FC $i$ at period $t$ (MW)

Submitted to the 21st Power Systems Computation Conference (PSCC 2020).

\begin{abstract}
$l_{j}^{G} \quad$ Linear cost coefficient of $\mathrm{MG} j(£ / \mathrm{MWh})$
$q_{j}^{G} \quad$ Quadratic cost coefficient of $\mathrm{MG} j\left(£ / \mathrm{MWh}^{2}\right)$

$g_{j}^{\max } \quad$ Maximum generation limit of MG $j$ (MW)

$s_{k}^{\max } \quad$ Power capacity of ES $k$ (MW)

$E_{k}^{\text {min }} \quad$ Minimum energy limit of ES $k(\mathrm{MWh})$

$E_{k}^{\max }$ Maximum energy limit of ES $k$ (MWh)

$E_{k}^{0} \quad$ Initial energy level of ES $k$ (MWh)

$\eta_{k}^{c} \quad$ Charging efficiency of ES $k$

$\eta_{k}^{d} \quad$ Discharging efficiency of ES $k$

C. Variables

$\lambda_{t}^{b} \quad$ Retail price for buying energy from the retailer at period $t$ (£/MWh)

$\lambda_{t}^{s} \quad$ Retail price for selling energy to the retailer at period $t$ (£/MWh)

$d_{i, t} \quad$ Demand of FC $i$ at period $t$ (MW)

$g_{j, t} \quad$ Generation of $\mathrm{MG} j$ at period $t$ (MW)

$s_{k, t}^{c} \quad$ Charging power of ES $k$ at period $t$ (MW)

$s_{k, t}^{d} \quad$ Discharging power of ES $k$ at period $t$ (MW)

$E_{k, t} \quad$ Energy level of ES $k$ at the end of period $t$ (MWh)

$u_{t} \quad$ Binary variable indicating whether the LEM buys energy from the retailer $\left(u_{t}=1\right)$ or sells energy to the retailer $\left(u_{t}=0\right)$ at period $t$

$n_{t} \quad$ Net demand of LEM at period $t\left(n_{t}>0\right.$ if the LEM buys energy from the retailer, $n_{t}<0$ if the LEM sells energy to the retailer) (MW)

$w_{t} \quad$ Net demand of retailer in the wholesale market at period $t\left(w_{t}>0\right.$ if the retailer buys energy from the wholesale market, $w_{t}<0$ if the retailer sells energy to the wholesale market) (MW)
\end{abstract}

\section{INTRODUCTION}

\section{A. Background and Motivation}

Although the ongoing decarbonization of energy systems promises to address environmental concerns, it also creates significant techno-economic challenges, mainly associated with the costly balancing of renewable generation and the increase of demand peaks driven by the electrification of transport and heat sectors [1]. A very promising paradigm change towards addressing these challenges lies in the deployment of flexible distributed energy resources (DER), including flexible loads, micro-generators and energy storage, which can support 
system balancing and reduce demand peaks [1]-[4]. However, this paradigm change greatly complicates the operation of the system, as the effective coordination of large numbers of small-scale DER involves very significant communication and computational scalability challenges as well as privacy concerns, since DER owners are not generally willing to disclose private information and be directly controlled by external entities [5].

One of the coordination approaches that has recently attracted great interest by both industry and academia is the establishment of local energy markets (LEM). Under this approach, the global coordination burden is broken down to the coordination of local market clusters, each grouping a number of end users with DER (lately referred to as community), and enabling direct energy trading between them [6]. Beyond addressing scalability and privacy challenges, the LEM concept exhibits a number of potential benefits, such as a) deferring or avoiding distribution network reinforcements as a result of matching local demand with local generation, b) enhancing the engagement of local end-users in system operation by creating a local identity and promoting social cooperation, and c) revitalizing the local economy by shaping opportunities for local investment, creating new jobs at the community level and promoting self-sufficiency. Recent studies have illustrated these benefits of LEM [7]-[11].

However, the introduction of LEM is also expected to have significant implications on the strategic interactions between the local end users and incumbent electricity retailers. After the deregulation of electricity systems, electricity retailers have been representing the large majority of the end users in the wholesale electricity market, by buying / selling energy consumed / produced by their end users from / to the wholesale market. The objective of these self-interested market entities lies in maximizing their individual profits by optimizing the retail prices offered to their customers but also respecting regulatory limits [12]. With the introduction of LEM, customers can directly trade energy between them, thus limiting their energy dependency on the incumbent retailer and increasing their economic surplus. This effect is expected to in turn impact the retailer's decisions regarding the offered prices and eventually its profit.

Numerous previous works have investigated the strategic interactions between retailers and individual customers with different types of DER [13]-[26]. Table I summarises in a structured way the main characteristics of these works. The considered types of DER include different combinations of flexible consumers (FC), micro-generators (MG) and energy storage (ES), with the majority of relevant works focusing on the former type. Furthermore, these papers generally adopt two different methodologies to model the retailer-customers interactions.

Specifically, authors in [13]-[17] employ single-level optimization models, which aim at maximising the retailer's profit; in these models, the optimal responses of the DER to the retail prices are expressed as closed-form functions. The limitation of this approach lies in the fact that the parameters of
TABLE I: Summary of Existing Literature Associated with the Examined Problem.

\begin{tabular}{cccc}
\hline Paper & Optimization model & DER types & Operation of LEM \\
\hline \hline$[13]$ & Single-level & FC & No \\
\hline$[14]$ & Single-level & FC & No \\
\hline$[15]$ & Single-level & FC & No \\
\hline$[16]$ & Single-level & FC, MG, ES & No \\
\hline$[17]$ & Single-level & FC, MG, ES & No \\
\hline$[18]$ & Bi-level (MPEC) & MG & No \\
\hline$[19]$ & Bi-level (MPEC) & FC & No \\
\hline$[20]$ & Bi-level (MPEC) & FC, MG, ES & No \\
\hline$[21]$ & Bi-level (iterative) & FC & No \\
\hline$[22]$ & Bi-level (iterative) & FC & No \\
\hline$[23]$ & Bi-level (MPEC) & FC, MG & No \\
\hline$[24]$ & Bi-level (MPEC) & FC, MG & No \\
\hline$[25]$ & Bi-level (MPEC) & FC & No \\
\hline$[26]$ & Bi-level (iterative) & FC & No \\
\hline This paper & Bi-level (non-convex) & FC, MG, ES & Yes \\
\hline & & &
\end{tabular}

these functions are determined based on exogenous data and therefore cannot accurately capture the impacts of the retail prices on the customers' response.

In order to address this limitation, authors in [18]-[26] employ bi-level optimization models in order to rigorously capture the interactions between the optimization of the pricing decisions of the self-interested retailer (modelled in the upper level - UL) and the optimization of the demand / supply response of its customers (modelled in the lower level - LL), which is thus represented endogenously and not based on exogenous data. These bi-level optimisation problems are solved either by converting them to Mathematical Programs with Equilibrium Constraints (MPEC) or by employing iterative algorithms (Table I).

As demonstrated in Table I, although the existing literature has investigated the strategic interactions between retailers and individual customers with different types of DER, the respective interactions between retailers and LEM integrating all these DER types and enabling energy trading between them, have not yet been analytically explored.

\section{B. Scope and Contributions}

This paper makes the first attempt to fill this knowledge gap by proposing a novel modelling approach capturing the interactions between the retailer's strategic pricing decisions and the operation of an LEM among its served customers. Specifically, this model is mathematically formulated as a multi-period bi-level optimization problem. The UL problem represents the strategic decision making of a self-interested retailer who determines the optimal time-specific retail prices offered to the customers for buying and selling energy so as to maximize its profit, while respecting regulatory constraints imposed on the offered retail prices. This UL problem is subject to four LL problems. The first three of them represent the decision making of $\mathrm{FC}, \mathrm{MG}$ and $\mathrm{ES}$, which do not participate in the LEM and thus optimize their demand / generation / storage response to the offered retail prices so as to maximize their own economic surplus. The fourth LL 
problem represents the operation of an LEM with different participants (FC, MG and ES) which, given the offered retail prices and the techno-economic parameters of its participants, determines the optimal dispatch of these participants and the energy exchanges with the retailer so as to maximize their total surplus.

Since this fourth LL problem is non-convex, as it includes the binary decision variables of the LEM to either buy or sell energy to the retailer at each period, the formulated bi-level optimization problem cannot be solved through the traditional approach of converting it to MPEC. In this context, this paper employs an approach recently proposed by the authors, which is based on the relaxation and primal-dual reformulation of the non-convex LL problem and the penalization of the associated duality gap [27].

The examined case studies comprehensively analyze the effects of introducing an LEM among the retailer's customers on the amount of energy served by the retailer, the buy and sell prices offered by the retailer, the retailer's profit and the different customers' economic surplus.

\section{Paper Structure}

The rest of this paper is organized as follows. Section II details the main assumptions, formulation and solution approach of the proposed bi-level optimization model. Case studies and quantitative results are presented in Section III. Finally, Section IV discusses conclusions and future extensions of this work.

\section{Proposed Model}

As discussed in Section I, the proposed model is mathematically formulated as a multi-period bi-level optimization problem with one UL and four LL problems, the structure of which is illustrated in Fig. 1.

\begin{tabular}{|c|c|c|c|c|c|c|c|c|}
\hline \multicolumn{9}{|c|}{$\begin{array}{l}\text { Max Profit of retailer } \\
\text { subject to: } \\
\text { > Regulatory constraints imposed on retail prices } \\
>\text { Balance between net energy traded with customers and }\end{array}$} \\
\hline $\begin{array}{c}\text { Buy } \\
\text { prices }\end{array}$ & $\begin{array}{l}\text { Demand } \\
\text { response }\end{array}$ & $\begin{array}{c}\text { Sell } \\
\text { prices }\end{array}$ & $\begin{array}{c}\text { Generation } \\
\text { response }\end{array}$ & $\begin{array}{l}\text { Buy prices } \\
\text { Sell prices }\end{array}$ & & & $\begin{array}{l}\text { Buy prices } \\
\text { Sell prices }\end{array}$ & $\begin{array}{c}\text { Net } \\
\text { response }\end{array}$ \\
\hline \multirow{2}{*}{\multicolumn{2}{|c|}{$\begin{array}{l}\text { LL1 problem } \\
\text { Independent FC } \\
\text { Max Demand utility } \\
\text { subject to: } \\
\text { > Demand operating } \\
\text { constraints }\end{array}$}} & \multirow{2}{*}{\multicolumn{2}{|c|}{\begin{tabular}{|l|} 
LL2 problem \\
Independent MG \\
Max Generation profit \\
subject to: \\
$>$ Generation \\
operating constraints
\end{tabular}}} & \multirow{2}{*}{\multicolumn{2}{|c|}{$\begin{array}{l}\text { LL3 problem } \\
\text { Independent ES } \\
\text { Max Storage profit } \\
\text { subject to: } \\
\text { > Storage operating } \\
\text { constraints }\end{array}$}} & \multicolumn{3}{|c|}{\begin{tabular}{|l|} 
LL4 problem \\
LEM operation
\end{tabular}} \\
\hline & & & & & & \multicolumn{3}{|c|}{\begin{tabular}{|l} 
Max LEM surplus \\
subject to: \\
$>$ Balance between local net energy \\
and net energy traded with retailer \\
$>$ Demand, generation, storage \\
operating constraints
\end{tabular}} \\
\hline
\end{tabular}

Fig. 1: Structure of proposed bi-level optimization model.

\section{A. Modelling Assumptions}

For clarity reasons, the main assumptions behind the proposed model are outlined below:

1) The proposed model expresses the decision making problem of a self-interested retailer which interacts with its customers and the wholesale market and aims at maximizing its overall profit.
2) For presentation clarity and without loss of generality, it is assumed that only one LEM operates among (a subset of) the retailer's customers; each customer can either participate in the LEM or trade independently with the retailer, neglecting the potential of customers participating directly in the wholesale market (which is a realistic assumption for small customers).

3) The retailer's customers (both the independent ones and the ones participating in the LEM) generally include flexible consumers, micro-generators and energy storage, each characterised by distinct operating properties.

4) Reflecting the reality, it is assumed that the examined retailer can offer differentiated prices to its customers for buying and selling energy, but these buy and sell prices are not differentiated for different customers to avoid discrimination concerns.

5) In contrast with the traditional fixed pricing or time-ofuse pricing regimes, where the offered retail prices (for both buying and selling energy) are flat throughout the examined daily horizon or during certain intervals of this horizon (e.g. peak and off-peak periods), the examined retailer can offer hour-specific retail prices to the served customers.

6) In order to prevent the retailer from exploiting the customers and making excessive profits, regulatory constraints are imposed on the minimum and maximum prices it can offer to its customers.

7) The examined retailer is assumed to serve a relatively small population of customers and therefore its decisions do not affect the wholesale prices, which are thus treated as fixed, exogenous parameters of the problem, i.e. the retailer is assumed to be a price-taker in the wholesale market.

\section{B. UL problem: Strategic Retailer}

The upper-level (UL) problem optimizes the pricing decisions of the examined retailer and is formulated as follows:

$$
\begin{array}{r}
\max _{\left\{\lambda_{t}^{b}, \lambda_{t}^{s}, w_{t}\right\}} \sum_{t} \lambda_{t}^{b}\left(\sum_{i} d_{i, t}+\sum_{k} s_{k, t}^{c}+u_{t} n_{t}\right)- \\
\sum_{t} \lambda_{t}^{s}\left(\sum_{j} g_{j, t}+\sum_{k} s_{k, t}^{d}+\left(u_{t}-1\right) n_{t}\right)-\sum_{t} \lambda_{t}^{w} w_{t}
\end{array}
$$

subject to:

$$
\begin{gathered}
\lambda^{\min } \leq \lambda_{t}^{b}, \lambda_{t}^{s} \leq \lambda^{\max }, \forall t \\
\sum_{i} d_{i, t}-\sum_{j} g_{j, t}+\sum_{k}\left(s_{k, t}^{c}-s_{k, t}^{d}\right)+n_{t}=w_{t}, \forall t
\end{gathered}
$$

The objective function (1a) maximizes the overall profit of the retailer, which includes the following components: i) its revenue from selling energy to demanding customers, including independent FC, independent ES when charging, and the LEM when buying energy from the retailer (first term), ii) its cost of buying energy from generating customers, including independent $\mathrm{MG}$, independent ES when discharging, and the LEM when selling energy to the retailer (second term); and iii) its net cost in the wholesale market, i.e. its cost / revenue of 
buying / selling energy from / to the wholesale market (third term).

The offered retail prices are subject to the regulatory constraints (1b) which aim at preventing the retailer from exploiting the customers and making excessive profits. Constraints (1c) express the energy balance constraints of the retailer; the net energy traded with its customers (including independent, FC, independent MG, independent ES and the LEM) and the net energy traded with the wholesale market are equal at each period.

\section{LL1 problem: Individual Flexible Consumers}

The first LL problem (LL1) optimizes the demand response of independent FC (not participating in the LEM) to the retail (buy) prices determined by the retailer, and is formulated as follows for independent $\mathrm{FC} i$ :

$$
\max _{\left\{d_{i, t}\right\}}\left(\sum_{t}\left(l_{i, t}^{D} d_{i, t}-q_{i, t}^{D} d_{i, t}^{2}\right)-\sum_{t} \lambda_{t}^{b} d_{i, t}\right)
$$

subject to:

$$
0 \leq d_{i, t} \leq d_{i, t}^{\max }, \forall t
$$

The objective function (2a) maximizes the utility of independent FC $i$, which is given by the difference between i) its benefit (or satisfaction) perceived from the use of energy (first term), modelled as a quadratic function of its demand levels; and ii) its cost of buying energy from the retailer (second term), modelled as the product of retail (buy) prices and its demand levels. Constraint (2b) expresses the flexibility of independent FC $i$ to modify its demand within certain limits.

\section{LL2 problem: Independent Micro-Generators}

The second LL problem (LL2) optimizes the generation response of independent MG (not participating in the LEM) to the retail (sell) prices determined by the retailer, and is formulated as follows for independent MG $j$ :

$$
\max _{\left\{g_{j, t}\right\}}\left(\sum_{t} \lambda_{t}^{s} g_{j, t}-\sum_{t}\left(l_{j}^{G} g_{j, t}+q_{j}^{G} g_{j, t}^{2}\right)\right)
$$

subject to:

$$
0 \leq g_{j, t} \leq g_{j}^{\max }, \forall t
$$

The objective function (3a) maximizes the profit of independent MG $j$, which is given by the difference between i) its revenue from selling energy to the retailer (first term), modelled as a quadratic function of its power outputs; and ii) its cost of production (second term), modelled as the product of retail (sell) prices and its power outputs. Constraint (3b) expresses the power output limits of independent MG $j$.

\section{E. LL3 problem: Independent Energy Storages}

The third LL problem (LL3) optimizes the charging / discharging response of independent ES (not participating in the LEM) to the retail (buy and sell) prices determined by the retailer, and is formulated as follows for independent ES $k$ :

$$
\max _{\left\{s_{k, t}^{c}, s_{k, t}^{d}, E_{k, t}\right\}}\left(\sum_{t} \lambda_{t}^{s} s_{k, t}^{d}-\sum_{t} \lambda_{t}^{b} s_{k, t}^{c}\right)
$$

subject to:

$$
\begin{gathered}
E_{k, t}=E_{k, t-1}+s_{k, t}^{c} \eta_{k}^{c}-s_{k, t}^{d} / \eta_{k}^{d}, \forall t \\
E_{k}^{\text {min }} \leq E_{k, t} \leq E_{k}^{\max }, \forall t \\
0 \leq s_{k, t}^{c} \leq s_{k}^{\max }, \forall t \\
0 \leq s_{k, t}^{d} \leq s_{k}^{\max }, \forall t \\
E_{k}^{0}=E_{k, N T}
\end{gathered}
$$

The objective function (4a) maximizes the profit of independent ES $k$, which is given by the difference between i) its revenue from selling energy to the retailer when discharging (first term) and ii) the cost of buying energy from the retailer when charging (second term). Constraint (4b) expresses the energy balance of the ES including charging and discharging losses. Constraint (4c)-(4e) represents its minimum and maximum energy and power limits. Finally, constraint (4f) expresses the energy neutrality assumption, i.e. the ES energy content at the start and the end of the examined horizon are assumed equal.

\section{F. LL4 problem: Local Energy Market}

Beyond individually contracted FC, MG and ES, the examined retailer trades energy with a LEM generally including the same types of customers and enabling energy trading between its participating customers. The fourth LL problem (LL4) represents the operation of the LEM which optimizes the dispatch of the participating customers and the energy exchanges with the retailer, and is formulated as follows:

$$
\begin{gathered}
\max _{V^{L L 4}}\left[\sum_{i^{\prime}, t}\left(l_{i^{\prime}, t}^{D} d_{i^{\prime}, t}-q_{i^{\prime}, t}^{D} d_{i^{\prime}, t}^{2}\right)-\sum_{j^{\prime}, t}\left(l_{j^{\prime}}^{G} g_{j^{\prime}, t}+q_{j^{\prime}}^{G} g_{j^{\prime}, t}^{2}\right)-\right. \\
\left.\sum_{t} \lambda_{t}^{b} u_{t} n_{t}+\sum_{t} \lambda_{t}^{s}\left(u_{t}-1\right) n_{t}\right]
\end{gathered}
$$

where:

$$
V^{L L 4}=\left\{d_{i^{\prime}, t}, g_{j^{\prime}, t}, s_{k^{\prime}, t}^{c}, s_{k^{\prime}, t}^{d}, E_{k^{\prime}, t}, u_{t}, n_{t}\right\}
$$

subject to:

$$
\begin{gathered}
\sum_{i^{\prime}} d_{i^{\prime}, t}-\sum_{j^{\prime}} g_{j^{\prime}, t}+\sum_{k^{\prime}}\left(s_{k^{\prime}, t}^{c}-s_{k^{\prime}, t}^{d}\right)=n_{t}, \forall t \\
u_{t} \in\{0,1\}, \forall t \\
0 \leq d_{i^{\prime}, t} \leq d_{i^{\prime}, t}^{\max }, \forall i^{\prime}, \forall t \\
0 \leq g_{j^{\prime}, t} \leq g_{j^{\prime}}^{\max }, \forall j^{\prime}, \forall t \\
E_{k^{\prime}, t}=E_{k^{\prime}, t-1}+s_{k^{\prime}, t}^{c} \eta_{k^{\prime}}^{c}-s_{k^{\prime}, t}^{d} / \eta_{k^{\prime}}^{d}, \forall k^{\prime}, \forall t \\
E_{k^{\prime}}^{\min } \leq E_{k^{\prime}, t} \leq E_{k^{\prime}}^{\max }, \forall k^{\prime}, \forall t \\
0 \leq s_{k^{\prime}, t}^{c} \leq s_{k^{\prime}}^{\max }, \forall k^{\prime}, \forall t \\
0 \leq s_{k^{\prime}, t}^{d} \leq s_{k^{\prime}}^{\max }, \forall k^{\prime}, \forall t \\
E_{k^{\prime}}^{0}=E_{k^{\prime}, N T}, \forall k^{\prime}
\end{gathered}
$$

The objective function (5a) maximizes the total surplus of the LEM, which includes the following components: i) the 
total benefit of all participating $\mathrm{FC} i^{\prime}$ (first term), ii) the total production cost of all participating MG $j^{\prime}$ (second term), iii) the cost of buying energy from the retailer when the LEM exhibits excess demand (third term), and iv) the revenue from selling energy to the retailer when the LEM exhibits excess generation (fourth term).

Constraints (5c) express the energy balance constraints of the LEM, ensuring that the excess demand / generation is bought from / sold to the retailer; the dual variables of these constraints constitute the LEM clearing prices. The fact that the LEM can either buy energy from the retailer or sell energy to the retailer at each period is expressed through the binary decision variable $u_{t}(5 \mathrm{~d})$. When the LEM exhibits excess demand $\left(n_{t}>0\right)$, it needs to buy energy from the retailer $\left(u_{t}=1\right)$, implying that the LEM incurs a cost to the retailer (as the third term of (5a) becomes positive) and does not earn a revenue from the retailer (as the fourth term of (5a) becomes zero). On the other hand, when the LEM exhibits excess generation $\left(n_{t}<0\right)$, it needs to sell energy to the retailer $\left(u_{t}=0\right)$, implying that the LEM earns a revenue from the retailer (as the fourth term of (5a) becomes positive) and does not incur a cost to the retailer (as the third term of (5a) becomes zero). Finally, constraints (5e)-(5k) express the individual operating constraints of the FC, MG and ES participating in the LEM, and follow the formulations adopted in problems (2), (3) and (4), respectively.

\section{G. Solution of Bi-Level Optimization Problem}

The five optimization problems illustrated in Fig. 1 and formulated in the previous sub-sections are coupled, since i) the buy and sell prices determined by the retailer (UL) affect the responses of the customers (as they constitute part of the objective functions of the LL1-LL4 problems), while ii) these responses affect the retailer's pricing decisions (as they constitute part of both the objective function and the energy balance constraints (1c) of the UL problem).

The traditional approach for solving such a bi-level optimization problem in the existing literature (see for example [18], [19], [21]-[24]) lies in converting it to a single-level MPEC. This is achieved by replacing the LL problems by their equivalent Karush-Kuhn-Tucker (KKT) optimality conditions, provided that the LL problems are continuous and convex, and introducing these conditions as constraints of the UL problem.

However, in the examined bi-level optimization problem, the fourth LL problem (LL4) representing the operation of the LEM, is non-convex as it includes the binary decision variables $u_{t}$ of the LEM to either buy energy from the retailer or sell energy to the retailer at each period (5d). This non-convexity prevents the derivation of equivalent KKT conditions for LL4 and thus means that the traditional MPEC approach is not applicable to the examined problem.

In order to address this fundamental challenge and solve the examined problem, this paper employs a new approach recently proposed by the authors in [27]. For space limitation reasons, we do not present the full details of this approach but only outline its main steps in Fig. 2 and below:

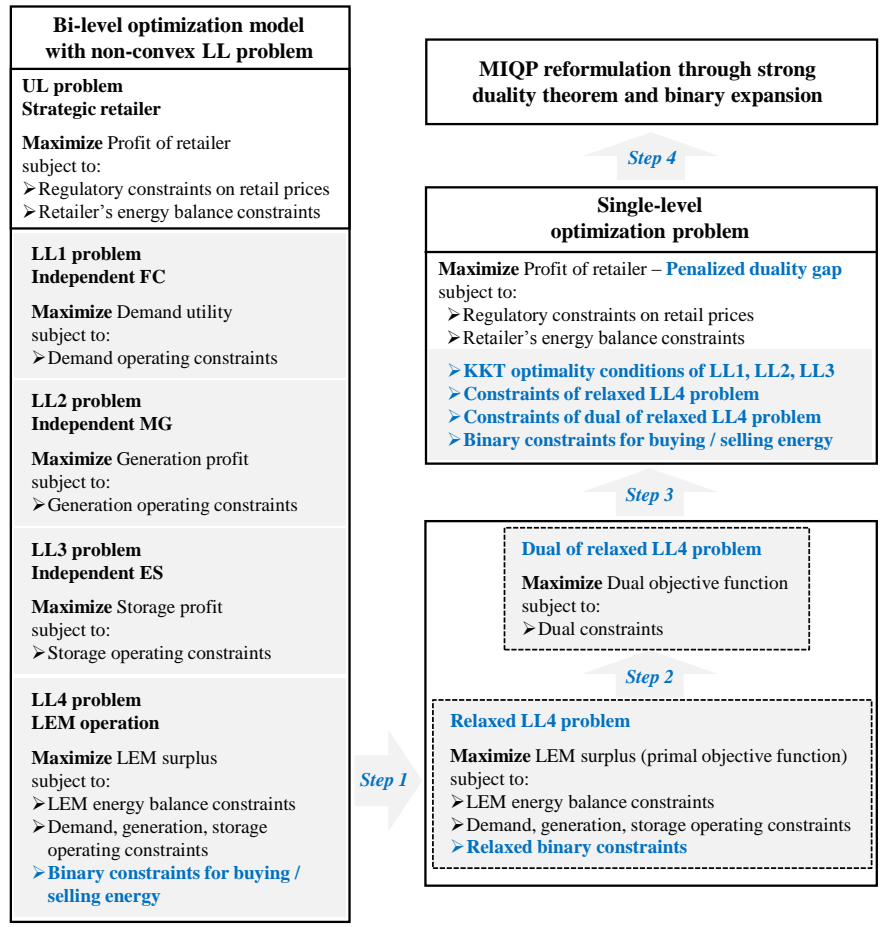

Fig. 2: Illustration of proposed solution approach.

Step 1: Problem LL4 is converted from a non-convex mixedinteger problem to a convex problem by relaxing its binary constraints (5d) as continuous constraints: $0 \leq u_{t} \leq 1, \forall t$.

Step 2: The dual problem associated with the relaxed LL4 problem is derived.

Step 3: A single-level problem is formulated which is subject to the constraints of the UL problem, the equivalent KKT optimality conditions of the three convex LL problems (LL1-LL3), the primal and dual constraints of the relaxed LL4 problem and the original binary constraints (5d). In order to ensure that the previous relaxation of LL4 does not substantially affect the solution optimality of this singlelevel problem, its objective function includes (apart from the objective function of the UL problem) a term penalizing the duality gap of the relaxed LL4 problem. The penalty value should be large enough to ensure that the single-level problem encapsulates a sufficiently accurate solution of the LL4 problem; on the other hand, an extremely large penalty value diminishes the importance of the retailer's profit in the objective function of this single-level problem, leading to inefficient pricing decisions by the retailer. Therefore, the penalty value should be suitably tuned, as further discussed in [27].

Step 4: Since this single-level problem is non-linear and cannot be efficiently solved by commercial solvers, we linearize it using a combination of techniques adopted in relevant literature, including the application of the strong duality theorem and binary expansion. The final problem is a mixed-integer quadratic program (MIQP) which can be efficiently solved to global optimality using commercial branch-and-cut solvers. 


\section{CASE STUdies}

\section{A. Test System, Examined Scenarios and Implementation}

The examined case studies apply the model developed in Section II in the context of a single day with hourly resolution, i.e. $T=\{1,2, . ., 24\}$. The examined retailer serves a set of customers including 12 aggregated FC of three different types, $6 \mathrm{MG}$ of three different types and 2 identical ES. The total generation capacity of the $6 \mathrm{MG}$ and the total power capacity of the $2 \mathrm{ES}$ are assumed to be equal to $50 \%$ and $10 \%$ of the aggregate peak demand of the 12 FC, respectively; these designing assumptions are made to ensure that a potential LEM among the retailer's customers can enable substantial amounts of energy trading between them, and examine conditions where the LEM can be both a buyer (during peak demand hours), but also a seller (during off-peak demand hours) of energy to the retailer. For space limitation reasons, the detailed operating parameters of these customers are provided in a supplementary document uploaded in Zenodo [28]. The assumed wholesale market prices across the examined day follow the pattern of a typical winter day in the UK. Finally, the maximum retail price is assumed $\lambda^{\max }$ $=250 £ / \mathrm{MWh}$.

The aim of the presented studies lies in analyzing the effects of introducing an LEM among the retailer's customers on both the retailer's and the customers' economic surplus. In this context, we examine three different scenarios regarding the composition of this LEM:

i) No LEM: This constitutes the benchmark scenario where an LEM does not exist and all customers interact individually with the retailer.

ii) Small LEM: Half of the retailer's customers (6 FC, 3 MG and 1 ES) participate in the LEM while the other half interacts individually with the retailer.

iii) Large LEM: All customers participate in the LEM.

The final MIQP model (Section II-G, Step 4) has been implemented and solved using the optimization software FICO $^{\mathrm{TM}}$ Xpress [29] on a computer with a 6-core $3.50 \mathrm{GHz}$ Intel(R) Xeon(R) E5-1650 v3 processor and 32 GB of RAM. The achieved optimality gap was lower than $0.02 \%$ in all the examined scenarios, and the average computational time across all the examined scenarios was $62.8 \mathrm{~s}$.

\section{B. Analysis of Retailer's Pricing Decisions and Customers' Response}

For each of the three examined scenarios, Fig. 3 illustrates the hourly profiles of a) the net demand of the LEM $n_{t}\left(n_{t}>0\right.$ if the LEM buys energy from the retailer, $n_{t}<0$ if the LEM sells energy to the retailer), b) the aggregate charging / discharging power of the 2 ES (positive values indicate charging and negative values indicate discharging), c) the total customers' demand served by the retailer $\left(\sum_{i} d_{i, t}+\sum_{k} s_{k, t}^{c}+\right.$ $\left.\left(u_{t}-1\right) n_{t}\right)$, and d) the total customers' generation served by the retailer $\left(\sum_{j} g_{j, t}+\sum_{k} s_{k, t}^{d}+\left(u_{t}-1\right) n_{t}\right)$. Furthermore, Fig. 4 illustrates the hourly profiles of a) the retail buy prices $\lambda_{t}^{b}$ offered by the retailer (and the wholesale prices $\lambda_{t}^{w}$ for comparison purposes), b) the retail sell prices $\lambda_{t}^{s}$ offered by the retailer (and the wholesale prices $\lambda_{t}^{w}$ for comparison purposes), c) the clearing prices of the LEM (in the two scenarios with LEM), which correspond to the dual variables of constraints (5c) (and the buy and sell prices offered by the retailer in the No LEM scenario for comparison purposes), and d) the comparison between the retail buy prices, retail sell prices and LEM clearing prices in the Small LEM scenario.

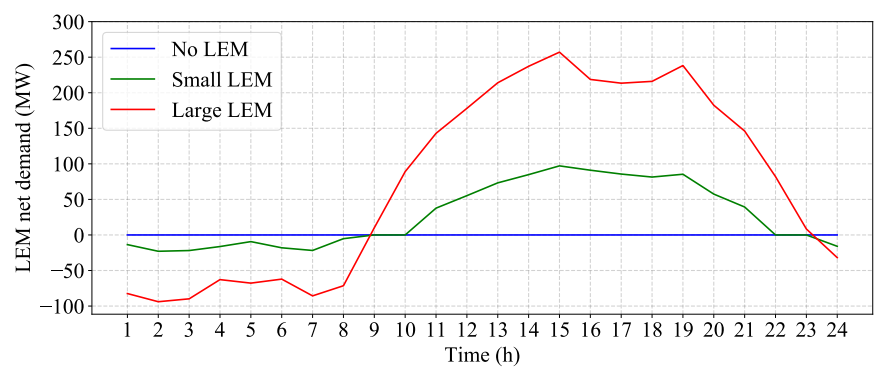

Fig. 3 (a): Net demand of LEM for different scenarios.

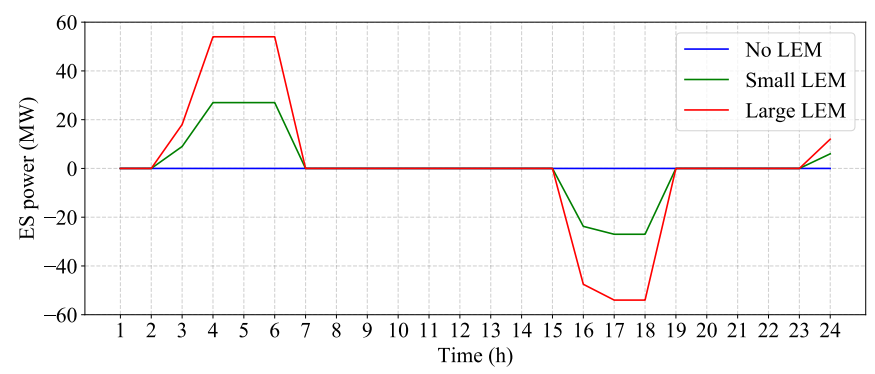

Fig. 3 (b): Aggregate charging / discharging power of all ES for different scenarios.

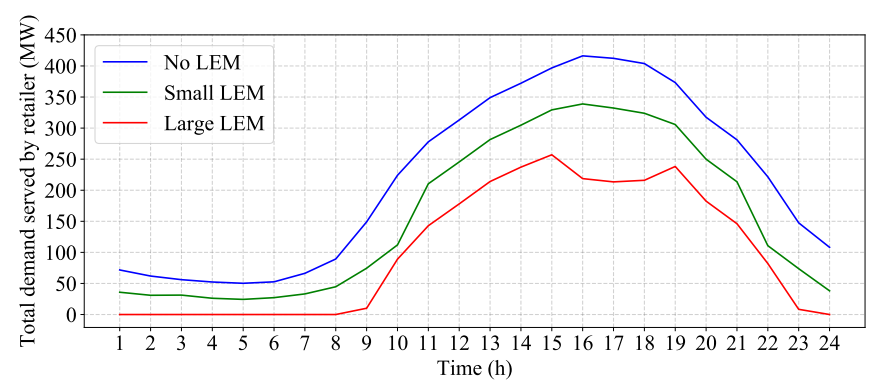

Fig. 3 (c): Total demand served by the retailer for different scenarios.

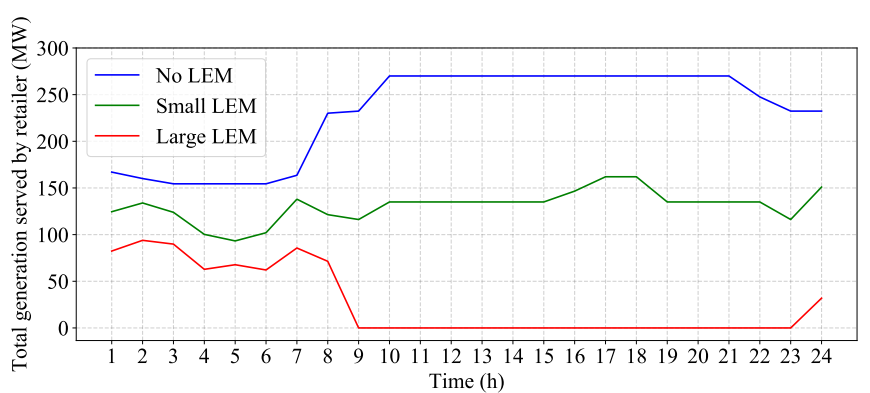

Fig. 3 (d): Total generation served by the retailer for different scenarios. 
In the benchmark scenario (No LEM scenario), both the total demand and the total generation served by the retailer exhibit the highest values across all hours (Fig. 3 (c) and 3 (d), respectively). This is because in the absence of an LEM, the only option for the customers to buy and sell energy is through the retailer. The strategic retailer exploits this dependency of its customers by offering very high buy prices (significantly higher than the wholesale prices) to demanding customers (Fig. 4(a)) and very low sell prices (significantly lower than the wholesale prices) to generating customers (Fig. 4(b); please note that, for clarity purposes, the high wholesale prices during hours 9-22 are not presented in this figure) in order to maximize its overall profit (1a). In other words, the strategic retailer exploits the customers by setting large differentials between buy and sell prices.

The offered buy prices are higher during peak hours, driven by a combination of the higher energy requirements of the retailer's consumers and the higher wholesale prices. The offered sell prices are also higher during peak hours, as the retailer strives to attract higher generation by its customers in order to reduce its net demand in the wholesale market which exhibits higher prices at these hours. However, even the lowest buy price (offered at hour 5) is higher than the highest sell price (offered at hours 10-21), rendering energy arbitrage nonprofitable. As a result, the ES served by the retailer prefer to remain idle (neither charge nor discharge throughout the day) in order to avoid negative profits (Fig. 3 (b)).

When an LEM is introduced (Small LEM and Large LEM scenarios), the participating customers have the additional option (apart from trading energy with the retailer) to trade energy between them. Given that the retailer offers very high buy prices and very low sell prices in the No LEM scenario, the LEM participants choose to exercise this option and trade energy at intermediate prices (Fig. 4(c)), which is mutually beneficial for all FC, MG and ES participants. As a result, the dependency of the LEM participants on the retailer is limited; both the total demand and the total generation served by the retailer are significantly reduced (Fig. 3(c) and 3 (d), respectively). It is worth noting however that although this dependency is reduced, it is not completely eliminated; the LEM still: i) buys energy from the retailer at peak hours, since its available generation and storage capacity is not sufficient to cover the high demand requirements during these hours, and ii) sells energy to the retailer at off-peak hours, since its available generation capacity is higher than the low demand requirements during these hours (Fig. 3 (a)).

Regarding the ES customers in particular, it is worth stressing that in these two scenarios, in contrast with the No LEM scenario, the ES participating in the LEM do not remain idle and carry out charging / discharging actions (Fig. 3 (b)), since such actions are beneficial for both: i) the ES participants themselves, given that they now face the LEM clearing prices which, in contrast to the retailer's differentiated buy and sell prices, render energy arbitrage profitable, as they are higher during peak hours and lower during off-peak hours (Fig. 4 (c)), and ii) the LEM as a whole, given that these actions further limit the dependency of the LEM on the retailer, as less demand is served by the retailer during peak hours (due to ES discharging) and less generation is served by the retailer during off-peak hours (due to ES discharging). In the same vein, it is noted that in the Small LEM scenario, the charging / discharging actions illustrated in Fig. 3 (b) correspond to the ES participating in the LEM while the other ES still remains idle.

As expected, this effect of limiting the dependency of the customers on the retailer is enhanced when all customers participate in the LEM (Large LEM scenario) with respect to the scenario where only half of them participate (Small LEM scenario). In particular, during the off-peak hours 24-8, the demand served by the retailer becomes zero (Fig. 3 (c)), as the available generation capacity is able to cover the low demand requirements; on the other hand, during the peak hours 9-23, the generation served by the retailer becomes zero (Fig. $3(\mathrm{~d})$ ), as the whole generation and storage capacity is utilized within the LEM to cover a part of the high demand requirements and limit as much as possible the dependency of the consumers on the retailer.

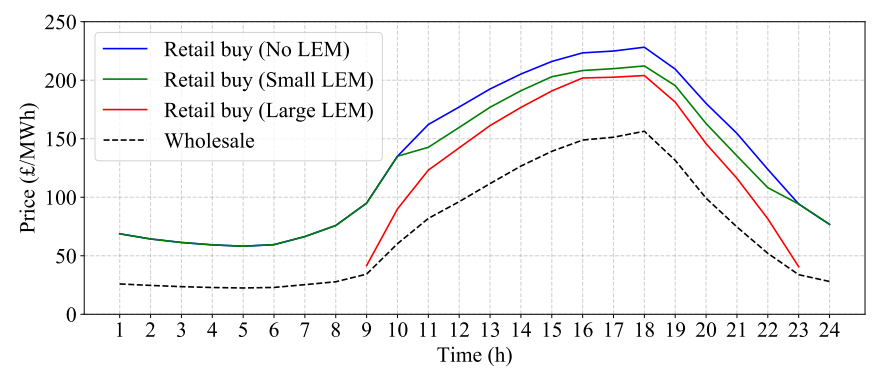

Fig. 4(a): Buy prices offered by the retailer for different scenarios.

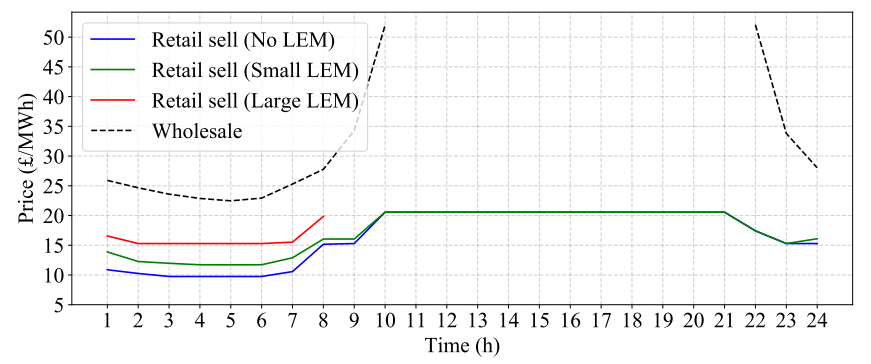

Fig. 4(b): Sell prices offered by the retailer for different scenarios.

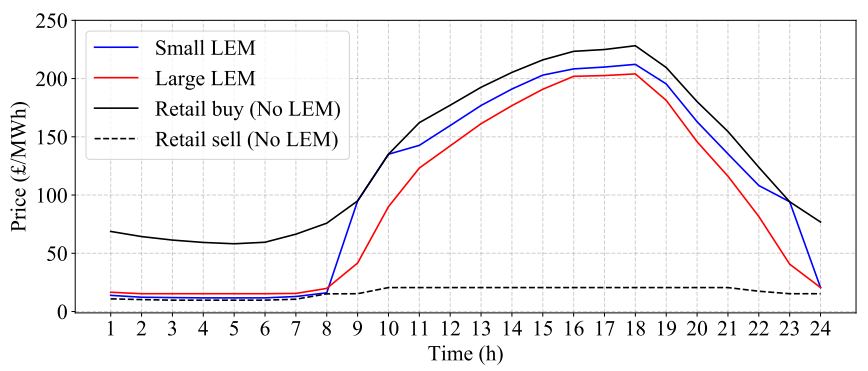

Fig. 4 (c): LEM clearing prices for different scenarios. 


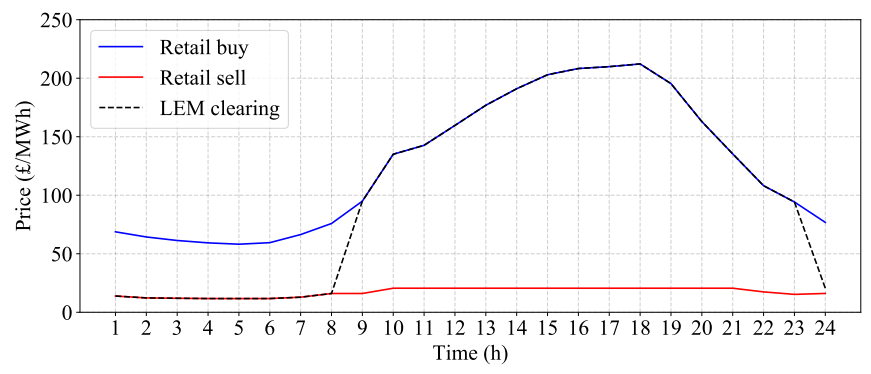

Fig. 4 (d): Buy prices, sell prices and LEM clearing prices in the Small LEM scenario.

As a result of this effect of LEM in limiting the dependency of the customers on the retailer, the latter reduces substantially the offered buy prices across the majority of hours (Fig. 4 (a)) in order to attract higher demand from its demanding customers and compensate the reduction of its served demand caused by the LEM. In mathematical terms, this is due to the fact that the revenue of the retailer (first term of its overall profit (1a)) depends on the summation of the buy price-demand products across all hours, i.e. the retailer needs to balance the trade-off between buy prices and customers' demand. In the Large LEM scenario and during the off-peak hours 24-8, given that the demand served by the retailer is zero (Fig. 3 (c)), the offered buy prices have no physical impact (in mathematical terms they constitute free variables) and thus are omitted from Fig. 4 (a).

In a similar spirit, the retailer increases substantially the offered sell prices across the majority of hours (Fig. 4 (b)) in order to attract higher generation from its generating customers and compensate the reduction of its served generation caused by the LEM. In mathematical terms, although this increase of the sell prices and customers' generation increases its cost at the retail side (second term of its overall profit (1a)), it reduces its net demand $w_{t}$ in the wholesale market (1c) and therefore reduces its cost (during peak hours) and increases its revenue (during off-peak hours) at the wholesale side (third term of its overall profit (1a)). In the Large LEM scenario and during the peak hours 9-23, given that the generation served by the retailer is zero (Fig. 3(d)), the offered sell prices have no physical impact (in mathematical terms they constitute free variables) and thus are omitted from Fig. 4 (b).

From a higher-level perspective, the above trends imply that the introduction of the LEM limits the retailer's strategic potential of exploiting the customers by setting large differentials between buy and sell prices. The offered buy prices are reduced and the offered sell prices are increased, i.e. they both move closer to the wholesale prices, implying that the retailer behaves more competitively.

It is also worth noting that in the Small LEM scenario and during off-peak hours, the LEM clearing prices are equal to the sell prices offered by the retailer (since the LEM sells its excess generation to the retailer), which are significantly lower than the buy prices offered at the same hours (Fig. 4(d)); this implies that FC participating in the LEM gain significant benefits with respect to individual FC during these hours. In a similar vein, during peak hours, the LEM clearing prices are equal to the buy prices offered by the retailer (since the LEM buys its excess demand from the retailer), which are significantly higher than the sell prices offered at the same hours; this implies that MG participating in the LEM gain significant benefits with respect to individual MG during these hours.

\section{Analysis of Retailer's and Customers' Economic Surplus}

Having analyzed the fundamental interactions between the retailer and the LEM through the demand / generation profiles of Fig. 3 and the price profiles of Fig. 4, the final part of this analysis lies in quantifying and analyzing the overall economic effects of the LEM on both the retailer and its customers. Starting from the former, Table II presents the total (daily) profit of the examined retailer and its three components (corresponding to the three terms of (1a)), for each of the examined scenarios.

TABLE II: Profit of Retailer for Different Scenarios.

\begin{tabular}{|c|c|c|c|c|}
\hline Scenario & Revenue (£) & Retail cost (£) & Wholesale net cost $(\mathfrak{f})$ & Profit $(£)$ \\
\hline No LEM & 913,313 & 96,288 & 93,645 & 723,380 \\
\hline$\overline{\text { Sma }}$ & 32 & 61,635 & 174,032 & 409,866 \\
\hline Large LEM & 402,109 & 10,522 & 271,815 & 119,772 \\
\hline
\end{tabular}

We start our analysis from the retailer's revenue which, as demonstrated in Table II, constitutes the most significant component of its overall profit. This revenue is greatly reduced in the Small LEM scenario (29\% with respect to the No LEM scenario) and further reduced in the Large LEM scenario (56\% with respect to the No LEM scenario). This trend is driven by the fact that the introduction of the LEM reduces both the total demand served by the retailer (Fig. 3(c)) and the buy prices offered by the retailer (Fig. 4(a)).

In a similar vein, the retailer's cost of buying energy from its generating customers is substantially reduced $(36 \%$ in the Small LEM scenario and $89 \%$ in the Large LEM scenario). This trend is driven by the fact that the introduction of the LEM reduces significantly the total generation served by the retailer (Fig. 3(d)), despite the relatively small increase of the sell prices offered by the retailer (Fig. 4 (b)).

Going further, the retailer's net cost in the wholesale market is significantly increased ( $86 \%$ in the Small LEM scenario and $190 \%$ in the Large LEM scenario). This trend is driven by the fact that the introduction of the LEM reduces the total generation served by the retailer to a higher extent than the reduction of the total demand served by the retailer (Fig. 3 (c) and $3(d)$ ), especially during peak hours when the difference between the LEM clearing prices and the sell prices offered by the retailer are extremely high (Fig. 4(c)). As a result, the retailer needs to buy more energy in the wholesale market, especially during peak periods which are characterized by high wholesale prices.

Overall, the introduction of the LEM reduces very significantly the retailer's total profit ( $43 \%$ in the Small LEM scenario and $83 \%$ in the Large LEM scenario), driven primarily 
by the reduction of its retail net revenue and secondarily by the increase of its wholesale net cost.

Moving our focus to the customers, Tables III-V present the total (daily) economic surplus of two identical FC, two identical MG and the two (identical) ES. The only difference between the two members of each of these sets of customers lies in the fact that, in the Small LEM scenario, the first member (the one corresponding to the second column of Tables III-V) participates in the LEM while the second (the one corresponding to the third column of Tables III-V) does not.

TABLE III: Utility of Flexible Consumers for Different Scenarios.

\begin{tabular}{|c|c|c|}
\hline Scenario & Utility of FC1 (£) & Utility of FC2 (£) \\
\hline No LEM & 32,890 & 32,890 \\
\hline Small LEM & 55,220 & 46,279 \\
\hline Large LEM & 70,327 & 70,327 \\
\hline
\end{tabular}

TABLE IV: Profit of Micro-generators for Different Scenarios.

\begin{tabular}{|c|c|c|}
\hline Scenario & Profit of MG1 (f) & Profit of MG2 (f) \\
\hline No LEM & 16,395 & 16,395 \\
\hline Small LEM & 154,956 & 20,266 \\
\hline Large LEM & 141,460 & 141,460 \\
\hline
\end{tabular}

TABLE V: Profit of Energy Storages for Different Scenarios.

\begin{tabular}{|c|c|c|}
\hline Scenario & Profit of ES1 (f) & Profit of ES2 (£) \\
\hline No LEM & 0 & 0 \\
\hline Small LEM & 14,991 & 0 \\
\hline Large LEM & 14,276 & 14,276 \\
\hline
\end{tabular}

The surplus of most customers is increased in the Small LEM scenario with respect to the No LEM scenario. This beneficial impact of the LEM is evident even for FC and MG not participating in the LEM, driven by the fact that the introduction of the LEM reduces the buy prices and increases the sell prices offered by the retailer (Fig. 4(a) and 4(b)). The only exception to this beneficial impact is ES2; in a similar fashion with the response of the two ES in the No LEM scenario, ES2 remains idle and thus makes zero profit (Table V), since the lowest buy price is still higher than the highest sell price, despite the above effects of the LEM (Fig. $4(\mathrm{~d})$ ).

However, this beneficial impact is significantly higher for customers participating in the LEM, since they trade energy based on the LEM clearing prices. As previously discussed, during off-peak hours, these prices are substantially lower than the buy prices offered by the retailer, creating additional benefits for FC1 participating in the LEM with respect to the individual FC2 (Table III). In a similar vein, during peak hours, the LEM clearing prices are significantly higher than the sell prices offered by the retailer, creating additional benefits for MG1 with respect to the individual MG2 (Table IV); it is worth noting that the surplus difference between MG1 and MG2 is significantly higher than the one between FC1 and
FC2, due to the extremely high difference between the LEM clearing prices and the retailer's sell prices during peak hours. Considering ES1 participating in the LEM, in contrast with the individual ES2, it does not remain idle and carries charging / discharging actions which generate positive profits (Table V), since it faces the LEM clearing prices which are higher during peak hours and lower during off-peak hours (Fig. 4(c)).

In the Large LEM scenario, the utility of $\mathrm{FC} 1$ is further increased with respect to the Small LEM scenario, since the LEM clearing prices are reduced during peak hours (Fig. 4(c)), driven by the participation of more generation and storage capacity in the LEM. On the other hand, this reduction of the LEM clearing prices during peak hours causes a reduction of the profit of MG1 and ES1 with respect to the Small LEM scenario. Finally, the surplus of FC2, MG2 and ES2 increases, since, in contrast with the Small LEM scenario, they now participate in the LEM and face the more favorable LEM clearing prices.

In order to summarize the above economic effects of the LEM on the retailer and its customers, Table VI presents the total economic surplus of the retailer, the total economic surplus of all FC, MG and ES (including the ones which participate in the LEM and the ones which do not), and the global economic surplus (or global welfare) which is defined as the sum of all entities' economic surpluses. As previously discussed, the introduction of the LEM reduces very significantly the retailer's economic surplus, while it increases significantly the economic surplus of all types of DER. The latter increase dominates the former reduction, implying that the introduction of the LEM results in an increase of the global welfare, i.e. in an enhancement of the overall efficiency of the market.

TABLE VI: Economic Surplus of Retailer and Customers for Different Scenarios.

\begin{tabular}{|c|c|c|c|c|c|}
\hline Scenario & Retailer $(£)$ & FC (£) & MG (£) & ES (£) & Global $(£)$ \\
\hline No I & 723,380 & 206,040 & 45,866 & 0 & 975,286 \\
\hline $\mathrm{Sm}$ & 409,866 & 328,390 & 321,761 & 14,991 & $1,075,008$ \\
\hline Large LEM & 119,772 & 423,394 & 542,761 & 28,552 & $1,114,479$ \\
\hline
\end{tabular}

\section{CONClusions AND Future Work}

This paper has explored for the first time the interactions between the operation of LEM with different types of participants (FC, MG and ES) and the strategic pricing decisions of incumbent electricity retailers, and has quantitatively analyzed the overall economic effects of LEM on both the retailer and its customers. In order to achieve that, this paper has developed a novel multi-period bi-level optimization model, which captures the pricing decisions of a retailer in the upper level (UL) problem and the response of both independent customers and the LEM in the lower level (LL) problems. Since the LL problem representing the LEM is non-convex, the traditional MPEC approach is not applicable for solving the developed bi-level problem, and a new approach recently proposed by the authors is employed instead, which is based on the relaxation 
and primal-dual reformulation of the non-convex LL problem and the penalization of the associated duality gap.

The presented case studies have provided numerous new and valuable insights around the role and impact of LEM. First of all, the introduction of an LEM is shown to reduce the customers' energy dependency on the retailer, since they are able to trade energy among them at prices which lie between the retailer's high buy prices and low sell prices, which is mutually beneficial for all FC, MG and ES participants; regarding the latter, the LEM is shown to unlock their arbitrage potential and activate them in the market. As a consequence, the retailer's strategic potential of exploiting the customers through large differentials between buy and sell prices is limited, and the retailer strives to make its offered buy and sell prices more competitive in order to attract more demand and generation by its customers. As a result of these effects, the profit of the retailer is very significantly reduced, while the customers enjoy significant economic benefits. Although this beneficial impact of LEM is significantly higher for customers participating in the LEM, it is also substantial for non-participating customers, due to the above effects of the LEM on the retailer's offered prices.

Future work aims at enhancing the developed model in two directions. First of all, the developed model assumes that the retailer's decisions do not affect the wholesale market prices, which constitutes a realistic assumption only for retailers serving a small population of customers. Future work aims at dropping this assumption and exploring the effects of the introduction of LEM on large retailers who can also act strategically in the wholesale market. Secondly, the presented model is deterministic, assuming that the examined retailer has accurate information around the operating characteristics of its customers and the operation of the LEM. Future work aims at incorporating realistic uncertainties that retailers face regarding these parameters and exploring the retailers' pricing decisions and profits in this setting.

\section{REFERENCES}

[1] A. Shakoor, G. Davies, and G. Strbac, "Roadmap for flexibility services to 2030," A report to the Committee on Climate Change. London: Pöyry, May 2017.

[2] I. J. Perez-Arriaga, "The transmission of the future: The impact of distributed energy resources on the network," IEEE Power and Energy Mag., vol. 14, no. 4, pp. 41-53, Jun. 2016.

[3] D. Pudjianto and G. Strbac, "Assessing the value and impact of demand side response using whole-system approach," Jour. Power and Energy, vol. 231, no. 6, pp. 498-507, Sep. 2017.

[4] D. Pudjianto, M. Aunedi, P. Djapic, and G. Strbac, "Whole-systems assessment of the value of energy storage in low-carbon electricity systems," IEEE Trans. Smart Grid, vol. 5, no. 2, pp. 1098-1109, Sep. 2013.

[5] D. Papadaskalopoulos, D. Pudjianto, and G. Strbac, "Decentralized coordination of microgrids with flexible demand and energy storage," IEEE Trans. Sustain. Energy, vol. 5, no. 4, pp. 1406-1414, Apr. 2014.

[6] European Commission, "Clean Energy for All Europeans". [Online]. Available: https://ec.europa.eu/energy/en/topics/energy-strategy-andenergy-union/clean-energy-all-europeans

[7] M. Gancheva, S. O'Brien, N. Crook, and C. Monteiro, "Models of local energy ownership and the role of local energy communities in energy transition in europe," European Committee of the Regions. Commission for the Environment, 2018.
[8] F. Teotia and R. Bhakar, "Local energy markets: Concept, design and operation," in Proc. 2016 Nat. Power Syst. Conf. (NPSC). IEEE, 2016, pp. 1-6.

[9] E. Mengelkamp, B. Notheisen, C. Beer, D. Dauer, and C. Weinhardt, "A blockchain-based smart grid: towards sustainable local energy markets," Comput. Sci. Res. Dev., vol. 33, no. 1-2, pp. 207-214, Feb. 2018.

[10] F. Lezama, J. Soares, P. Hernandez-Leal, M. Kaisers, T. Pinto, and Z. Vale, "Local energy markets: Paving the path toward fully transactive energy systems," IEEE Trans. Power Syst., vol. 34, no. 5, pp. 4081-4088, Sep. 2018.

[11] P. Siano, G. De Marco, A. Rolán, and V. Loia, "A survey and evaluation of the potentials of distributed ledger technology for peer-to-peer transactive energy exchanges in local energy markets," IEEE Syst. J., vol. 13, no. 3, pp. 3454-3466, Sep. 2019.

[12] J. Yang, J. Zhao, F. Luo, F. Wen, and Z. Y. Dong, "Decision-making for electricity retailers: A brief survey," IEEE Trans. Smart Grid, vol. 9 no. 5, pp. 4140-4153, Jan. 2017.

[13] J. Yusta, I. Ramirez-Rosado, J. Dominguez-Navarro, and J. Perez-Vidal, "Optimal electricity price calculation model for retailers in a deregulated market," Int. J. Elect. Power Energy Syst., vol. 27, no. 5-6, pp. 437-447, Jun.-Jul. 2005.

[14] P. Faria and Z. Vale, "Demand response in electrical energy supply: An optimal real time pricing approach," Energy, vol. 36, no. 8, pp. 53745384, Aug. 2011.

[15] M. Doostizadeh and H. Ghasemi, "A day-ahead electricity pricing model based on smart metering and demand-side management," Energy, vol. 46, no. 1, pp. 221-230, Oct. 2012.

[16] S. Nojavan, K. Zare, and B. Mohammadi-Ivatloo, "Optimal stochastic energy management of retailer based on selling price determination under smart grid environment in the presence of demand response program," Appl. Energy, vol. 187, pp. 449-464, Feb. 2017.

[17] S. Nojavan and K. Zare, "Optimal energy pricing for consumers by electricity retailer," Electr. Power Energy Syst., vol. 102, pp. 401-412, Nov. 2018.

[18] J. M. López-Lezama, A. Padilha-Feltrin, J. Contreras, and J. I. Muñoz, "Optimal contract pricing of distributed generation in distribution networks," IEEE Trans. Power Syst., vol. 26, no. 1, pp. 128-136, Feb 2010.

[19] M. Zugno, J. M. Morales, P. Pinson, and H. Madsen, "A bilevel model for electricity retailers' participation in a demand response market environment," Energy Econ., vol. 36, pp. 182-197, May 2013.

[20] G. E. Asimakopoulou, A. G. Vlachos, and N. D. Hatziargyriou, "Hierarchical decision making for aggregated energy management of distributed resources," IEEE Trans. Power Syst., vol. 30, no. 6, pp. 3255-3264, Nov. 2015.

[21] M. Yu and S. H. Hong, "Supply-demand balancing for power management in smart grid: A stackelberg game approach," Appl. Energy, vol. 164, pp. 702-710, Feb. 2016.

[22] S. Sekizaki, I. Nishizaki, and T. Hayashida, "Electricity retail market model with flexible price settings and elastic price-based demand responses by consumers in distribution network," Int. J. Electr. Power Energy Syst., vol. 81, pp. 371-386, Oct. 2016.

[23] S. Bahramara, M. P. Moghaddam, and M. Haghifam, "A bi-level optimization model for operation of distribution networks with microgrids," Electr. Power Energy Syst., vol. 82, pp. 169-178, Nov. 2016.

[24] G. E. Asimakopoulou and N. D. Hatziargyriou, "Evaluation of economic benefits of der aggregation," IEEE Trans. Sustain. Energy, vol. 9, no. 2, pp. 499-510, Aug. 2017.

[25] C. Zhang, Q. Wang, J. Wang, P. Pinson, J. M. Morales, and J. Østergaard, "Real-time procurement strategies of a proactive distribution company with aggregator-based demand response," IEEE Trans. Smart Grid, vol. 9, no. 2, pp. 766-776, Mar. 2018.

[26] R. Lu, S. H. Hong, and X. Zhang, "A dynamic pricing demand response algorithm for smart grid: reinforcement learning approach," Appl. Energy, vol. 220, pp. 220-230, Jun. 2018.

[27] Y. Ye, D. Papadaskalopoulos, J. Kazempour, and G. Strbac, "Incorporating non-convex operating characteristics into bi-level optimization electricity market models," IEEE Trans. Power Syst., vol. 35, no. 1, pp. 163-176, Jan. 2020.

[28] Zenodo datasheet. [Online]. Available: http://doi.org/10.5281/zenodo.3742998

[29] F. XPRESS. [Online]. Available: http://www.fico.com/en/Products/DMTools/ Pages/FICO-XpressOptimization-Suite.aspx 